\section{Kurzbericht über die ordentliche Ärzte- kammersitzung vom 21. und 22. Juni 2000 in Bern}

\author{
F.-X. Deschenaux, FMH-Generalsekretär
}

Diese Ärztekammersitzung zeichnete sich ganz besonders dadurch aus, dass es die erste war, die in neuer Zusammensetzung gemäss neuen FMH-Statuten zusammentrat.

Die Sitzung bescherte den 200 versammelten Delegierten eine fast gigantische anmutende Tagesordnung, mit 89 vor Tagungsbeginn eingegangenen Anträgen, mit, neben den statutarischen Geschäften (Jahresbericht, Jahresrechnung 1999 sowie Budget 2001), den Schwerpunkten: Wahlen für die Amtsperiode 2000-2003, Neuordnung der Facharzttitel und Weiterbildungsprogramme sowie ausführliche Beratungen rund um den Kontrahierungszwang und die neusten Entwicklungen in Sachen TarMed. Gerade die beiden letzten Themen haben, wie gut vorstellbar, im Lichte der jüngsten, bestimmt nicht zufälligerweise zu diesem Zeitpunkt erfolgten, Interventionen des Eidg. Departementes des Innern und dessen Vorsteherin, eine ganz besondere Resonanz erzielt.

Die Beschlüsse werden im vorliegenden Bericht in der Reihenfolge gemäss Traktandenliste wiedergegeben.

\section{Mitteilungen und Bestellung des Büros}

Die Sitzung wird um 9.45 Uhr eröffnet, nachdem das erforderliche Quorum von 100 Delegierten bereits weit überschritten ist.

Die vom Präsidenten vorgeschlagenen Stimmenzähler für den ersten Sitzungstag (Dres. A. Sury, A. Bacchetto, A. Froidevaux, C. Ehrler und M. Hug) werden stillschweigend genehmigt; für den zweiten Sitzungstag wurden zu Beginn der Sitzung vorgeschlagen und stillschweigend genehmigt: Dres. Z. Schneider, C. Ehrler, A. Kubli Bauer, M. Schibli, M. Kondo Oestreicher und C. Brunner.

Das Wahlbüro, welches mit der Auszählung der Stimmzettel für die Gesamterneuerungswahlen 2000-2003 beauftragt war, setzte sich aus Mitarbeiterinnen und Mitarbeitern des Generalsekretariats unter der Leitung von Herrn C. Kreyden zusammen, damit die als Stimmenzähler fungierenden Delegierten nicht von den während der Auszählung der Stim- men zu fassenden Beschlüssen ausgeschlossen wurden. Dieses Vorgehen entspricht einer gut eingeführten Tradition, die von den Delegierten nicht in Frage gestellt wird.

Der Präsident H. H. Brunner präzisiert zuhanden des Protokolls die ab jetzt und in Zukunft geltende Regelung für die Begleitpersonen. Die Basisorganisationen (Kantonale Ärztegesellschaften [nachfolgend KG], Verband Schweizerischer Assistenz- und Oberärztinnen und -ärzte [VSAO]) waren bis anhin befugt gewesen, sich von einem nichtärztlichen Berater begleiten zu lassen, der auch das Rederecht besass, sofern ihm dieses nicht durch einen Ordnungsantrag entzogen wurde. Diese Usanz wird aufrecht erhalten, aber der Zentralvorstand (ZV) bzw. der Präsident behalten sich das Recht vor, zu verlangen, dass, wegen der durch die grosse Anzahl Delegierter eingeschränkten Platzverhältnisse, allfällige nichtärztliche Begleitpersonen von medizinischen Fachgesellschaften (nachfolgend: FG) nicht im Plenarsaal, sondern auf der Galerie Platz nehmen. Daneben behält sich der ZV das Recht vor, zur Behandlung besonderer Geschäfte aussenstehende Personen, deren Anwesenheit sich als wichtig erweist, zu den Ärztekammersitzungen einzuladen.

Aufgrund der beeindruckenden Traktandenliste dieser Ärztekammer, lässt der Präsident verlauten, dass die Sitzung am ersten Tag voraussichtlich bis etwa 18.30 Uhr dauern, und das Sitzungsende des zweiten Tages von der Durchberatung der Traktandenliste abhängig gemacht wird.

Gemäss der neuen Geschäftsordnung der FMH vom 8. April 1999, die sich ihrerseits auf Art. 35 Abs. 2 der neuen Statuten bezieht, wird künftig nur noch ein Beschlussprotokoll geführt, das hier und dort, sofern notwendig, durch einige zusätzliche Erläuterungen ergänzt wird.

Der Präsident stellt weiter fest, dass die Einladung mit der Traktandenliste, eingeschlossen die Veröffentlichung in der Schweizerischen Ärztezeitung (nachfolgend: SÄZ), fristgerecht erfolgt ist. Sämtliche bis Montagabend eingetroffenen Anträge sind übersetzt worden.

Auf Einladung des Präsidenten, erläutert der Geschäftsführer der FMH Services, Herr M. Baumgartner, dass die neuen Statuten der FMH Services neu auch Nicht-FMH-Mitgliedern das Recht zugestehen, dieser Genossenschaft anzugehören, weshalb kein Grund mehr besteht, die traditionsgemäss vorgängig der ordentlichen Ärztekammer durchgeführte Delegiertenversammlung der FMH Services weiterhin stattfinden zu lassen. Die Delegiertenversammlung der FMH Services wird künftig durch eine Universalversammlung ersetzt, die ihre Beschlüsse in einer Urabstimmung fällt. Die Mitglieder werden das Stimmmaterial nächstens erhalten.

Der Präsident schliesst dieses erste Traktandum mit einem Nachruf auf die im laufenden Geschäftsjahr verstorbenen Kolleginnen und Kollegen. Es liegt ihm besonders daran, Dr. Jürg Ammann, des kürzlich verstorbenen Präsidenten der FMS, zu gedenken und dessen grosse Verdienste herauszustreichen, die die- 
ser sich mit seinem ausserordentlichen Engagement für die Sache seiner chirurgisch tätigen Kolleginnen und Kollegen erworben hat. Sein Kampf war weit entfernt von einer reinen Verteidigung der Honorare, denn seine Aktivitäten zielten viel höher und standen in Zusammenhang mit seinen Anstrengungen, der Abwertung des beruflichen Status Einhalt zu gebieten. In dieser Hinsicht gebührt ihm der Dank der gesamten Ärzteschaft.

Auf Ersuchen des Präsidenten erhebt sich die Kammer zu einer Schweigeminute im Gedenken an alle verstorbenen Mitglieder.

\section{Jahresbericht $1999 / 2000$}

Der Jahresbericht, erstellt durch den Präsidenten der FMH, die Ressortverantwortlichen im ZV, den Generalsekretär und die verschiedenen Leiterinnen und Leiter von Dienstbereichen und Abteilungen im Generalsekretariat, ist in der SÄZ Nr. 23 vom 7. Juni 2000 publiziert worden.

Der Präsident ergänzt den Bericht durch ein paar einerseits rückblickenden und auf die mit dem heutigen Tag beginnende neue Amtsperiode vorausschauenden Bemerkungen.

Das Wort wird nach dieser vom Präsidenten vorgenommenen Übersicht nicht verlangt und der Jahresbericht wird ohne Gegenstimmen genehmigt.

\subsection{Jahresrechnung 1999 der FMH}

\subsubsection{Genehmigung}

Bilanz und Erfolgsrechnung der FMH für das Jahr 1999 sowie der beigefügte Revisionsbericht der Kontrollstelle PriceWaterhouseCoopers werden fast einstimmig, mit einer Enthaltung, genehmigt. Der Ausgabenüberschuss von Fr. 77 744.98, der um etwa Fr. 429 000.- wesentlich niedriger ausgefallen ist als budgetiert, geht zu Lasten des Kontos Eigenkapital, welches damit per 31. Dezember 1999 einen Stand von Fr. 4450873.69 aufweist.

Anschliessend werden Bilanz und Erfolgsrechnung per 31. Dezember 1999 des Hilfsfonds sowie Bilanz und Erfolgsrechnung per 31. Dezember 1999 des Fonds zur Förderung der Weiterbildung von Schweizer Ärzten in Entwicklungsländern gemeinsam genehmigt.

\subsubsection{Déchargeerteilung an den Zentralvorstand}

Den verantwortlichen Organen wird mit überwältigender Mehrheit, bei einer Enthaltung, Décharge erteilt.

Antrag zu Traktandum Nr. 3.1 von Dr. R. Streit, Ärztegesellschaft des Kantons Bern

Der Antrag, welcher durch Dr. M. Battaglia, namens des VSA0, vollumfänglich unterstützt wird, hat folgenden Wortlaut: «Der Zentralvorstand wird beauftragt, an der ordentlichen Ärztekammer 2001, gestützt auf die Kostenstellenrechnung, aufzuzeigen, wie Kosten und Erträge im Bereiche der einzelnen
Kostenstellen anfallen. Das beschäftigte Personal pro Kostenstelle ist anzugeben.»

Der Präsident H. H. Brunner nimmt namens des Zentralvorstands diesen Antrag entgegen.

\subsection{Budget 2001 der FMH}

Das der Ärztekammer vorliegende Budget für das Jahr 2001 enthält zwei Teile: einerseits das allgemeine Budget, welches bei Einnahmen von insgesamt Fr. 12755 000.- und Ausgaben von total Fr. 12684 000.einen Einnahmenüberschuss von Fr. $71000 .-$ vorsieht, und andererseits zeitlich befristete Sonderbeiträge zur Finanzierung besonderer Aktivitäten. Das vorliegende Budget basiert auf einem Zentralbeitrag von Fr. 480.- für die Mitglieder der Kategorien 1 (selbständig tätige Ärztinnen und Ärzte) und 2 (unselbständig tätige Ärztinnen und Ärzte in leitender Funktion), von Fr. 240.- für Mitglieder der Kategorien 3 (unselbständige Ärztinnen und Ärzte nicht in leitender Funktion und nicht in FMH-Weiterbildung) und 4 (Ärztinnen und Ärzte in FMH-Weiterbildung) sowie von Fr. 120.- für Mitglieder der Kategorien 5 (Ärztinnen und Ärzte mit Wohnsitz und Berufstätigkeit im Ausland) und 6 (vorübergehend nicht als Ärztin oder Arzt berufstätige Mitglieder).

Auf stillschweigende Genehmigung des Antrags des Zentralvorstandes hin, wird über das allgemeine Budget und über die Sonderbeiträge getrennt abgestimmt.

Das allgemeine Budget gibt zu keinen Diskussionen Anlass und wird bei einer Gegenstimme und 3 Enthaltungen angenommen.

Die Sonderbeiträge hingegen werden ausführlich diskutiert. Wir geben hier eine Zusammenfassung der Beschlüsse wieder. Der ursprünglich vom ZV vorgesehene Gesamtbetrag der Sonderbeiträge betrug Fr. 120.- für alle FMH-Mitglieder und war zur Finanzierung folgender Aufgaben vorgesehen: Schweizerische Arzneimittelnebenwirkungszentrale: Fr. 5.zum letzten Mal im Jahr 2001; einmaliger Sonderbeitrag von Fr. 20.- für das Jahr 2001 zur Finanzierung des Ausbaus des Verlages EMH; «HIN-Abonnement für alle», geltend für die Jahre 2001, 2002 und 2003, von max. Fr. 50.-; "Praxisassistenz" Fr. 25.- für die Mitgliederkategorien 1-4 und schliesslich Fr. 20.- für die Schlussarbeiten und die Einführung von TarMed, geltend für die Mitgliederkategorien 1, 2, 3 und 4.

Gegenüber denjenigen, die wie Dr. R. Streit (BE) vorgezogen hätten, dass man die notwendigen Mittel beantragt und sie in das allgemeine Budget integriert, rechtfertigt der Präsident die Sonderbeiträge mit dem Argument einer besseren Transparenz und einer Zusammenfassung der Finanzierung von zeitlich befristeten Aufgaben. Die Vertreter des VSAO (Dres. M. Battaglia und S. Stöhr), obwohl zurückhaltend gegenüber einigen Geschäften (insbesondere HIN), stellen sich nicht grundsätzlich gegen das Prinzip von Sonderbeiträgen, beantragen jedoch, dass diese besser abgestuft werden, analog der Abstufungen der verschiedenen Mitgliederkategorien. Die Finanzierung dieser Aufgaben wird also von nieman- 
dem bestritten, einige Delegierte setzen sich sogar ganz ausdrücklich dafür ein, insbesondere für HIN.

Der Präsident unterbreitet danach namens des Zentralvorstands einen Kompromissantrag. Er beantragt die Erhebung eines Sonderbeitrages von Fr. 100.- entweder für alle (Variante I) oder von Fr. 70.für Beitragskategorien 3 und 4 und Fr. 110.- für die Kategorien 1 und 2 (Variante II). Die angenommene Variante wird anschliessend in der Hauptabstimmung dem Antrag des VSAO gegenübergestellt. Diesem Vorgehen wird zugestimmt und Dr. P.-A. Schneider von der Ärztegesellschaft des Kantons Genf/AMG, welcher beantragt hatte, dass der Beitrag für die Praxisassistenz von Fr. 25.- auf Fr. 10.- reduziert werde, zieht seinen Antrag daraufhin zurück. Der Präsident präzisiert vor der Abstimmung, dass die Verteilung der im Rahmen dieser Sonderbeiträge erhobenen Summe auf die einzelnen Bereiche durch Beschluss des Zentralvorstands vorgenommen werden.

In einer knapp ausgehenden ersten Abstimmung überwiegt Variante I gegenüber Variante II mit $76 \mathrm{zu}$ 75 Stimmen. In einer zweiten Abstimmung, in welcher Variante I dem Antrag des VSAO gegenübergestellt wird, siegt die Variante I mit 117 Stimmen, 49 Gegenstimmen und 3 Enthaltungen.

Bevor dieses Traktandum geschlossen wird, sei noch der Vorschlag des Präsidenten der Ärztegesellschaft des Kantons St. Gallen, Dr. R. Urscheler, erwähnt: Er wünscht, dass an der nächsten Präsidentenkonferenz unter den Kantonalen Ärztegesellschaften nochmals eine Umfrage betreffend der Fakturierung der FMH-Zentralbeiträge durchgeführt wird, um herauszufinden, ob die Fakturierung durch die KGs oder das FMH-Generalsekretariat erfolgen soll. Dem Wunsch wird entsprochen werden.

\section{Wahlen für die neue Amtsperiode 2000-2003}

4.1 Festlegung der Anzahl Mitglieder des Zentralvorstandes für die nächste Amtsperiode

$\mathrm{Zu}$ dieser Frage liegen einander konkurrierende Anträge vor. Auf der einen Seite unterbreiten der VSA0 und die Ärztegesellschaft des Kantons Basel-Stadt Anträge auf Beibehaltung des Zentralvorstands mit 11 Mitgliedern. Auf der andern Seite hatte Dr. A. Haefeli, Präsident des VEDAG, ursprünglich einen Antrag eingereicht, der verlangte, dass die beiden im ZV freigewordenen deutschschweizerischen Sitze nicht mehr besetzt würden, dies im Sinne eines Moratoriums. Dr. Haefeli zieht jedoch seinen Antrag im Laufe der Verhandlungen zurück, weil gemäss Statuten ein Sitz dem Verein der Leitenden Spitalärzte der Schweiz/VLSS zusteht und weil sogar jene, die heute für einen ZV von 11 Mitgliedern einstehen, sich grundsätzlich einer künftigen Reduktion nicht widersetzen, sofern der ZV im Jahre 2001 ein neues Führungskonzept für die FMH vorlegt. Dr. A. Haefeli schliesst sich vorbehaltlos dem unter Traktandum 5 eingereichten Antrag von Dr. R. Streit an und erklärt, dass der Teil des VEDAG-Antrages, soweit er nicht das Moratorium für die neue Amtsperiode betrifft, unter
Traktandum 5 Statutenänderung behandelt werden soll.

Andere Delegierte unterstützen eine sofortige Reduktion des ZV auf 9 Mitglieder. Diese zwei unterschiedlichen Meinungen müssen zunächst einander gegenübergestellt werden. In der Abstimmung beschliessen die Delegierten mit 125 gegen 48 Stimmen und 4 Enthaltungen, für die Amtsperiode 2000-2003 die Zusammensetzung des ZV mit 11 Mitgliedern beizubehalten.

\subsection{Wahl des Zentralvorstandes der FMH}

Bestätigung der bisherigen Mitglieder

Eingegangene Stimmzettel $\quad 176$

Gültige Stimmzettel 173

Absolutes Mehr 87

Wiedergewählt sind:

VEDAG

Dr. Hans Heinrich Brunner (LU) 165 Stimmen

Dr. Max Giger (ZH)

Dr. Reto Laetsch (SG)

Dr. Ursula Steiner-König (BE)

150 Stimmen

156 Stimmen

155 Stimmen

\section{SMSR}

Dr. Claude Aubert (GE)

Dr. Yves Guisan (VD)

166 Stimmen

152 Stimmen

Dr. Marie-Christine Peter-Gattlen (VS) 158 Stimmen

VSA0

Dr. Ludwig-Theodor Heuss (BS)

167 Stimmen

Wahl für die vakanten Sitze

ORDINE (OMCT)

Eingegangene Stimmzettel

175

Gültige Stimmzettel

174

Absolutes Mehr

88

Gewählt ist:

Dr. Franco Muggli (TI)

174 Stimmen

\section{VLSS}

Eingegangene Stimmzettel $\quad 172$

Gültige Stimmzettel $\quad 168$

Absolutes Mehr $\quad 85$

Gewählt ist:

Dr. Verena Briner, PD (LU)

94 Stimmen

Weiter haben Stimmen erhalten:

Dr. Yves Guisan (VD)

Prof. Werner Straub (BE)

Übrige

58 Stimmen

15 Stimmen

1 Stimme

\section{Restlicher Sitz}

Eingegangene Stimmzettel $\quad 174$

Gültige Stimmzettel 161

Absolutes Mehr

Gewählt ist:

Dr. Susanna Stöhr (BS)

83 Stimmen 
Weiter haben Stimmen erhalten:

Dr. Catherine Wehren (BS)

Dr. Ludwig-Theodor Heuss (BS)

76 Stimmen

2 Stimmen

4.3 Wahl des Präsidenten der FMH

Eingegangene Stimmzettel

Gültige Stimmzettel

166

Absolutes Mehr

Gewählt ist:

Herr Dr. Hans Heinrich Brunner

152 Stimmen

Weiter haben Stimmen erhalten:

Herr Dr. Claude Aubert

3 Stimmen

Herr Dr. Yves Guisan

Herr Dr. Ludwig-Theodor Heuss

5 Stimmen

3 Stimmen

\subsection{Wahl der beiden Vizepräsidenten der FMH}

\section{Offene Wahl}

Auf Anregung aus der Versammlung, lässt der Präsident zunächst darüber abstimmen, ob von diesem Moment an die folgenden Wahlen offen durchgeführt werden sollen. Die Delegierten stimmen diesem Vorgehen mit 147 gegen 19 Stimmen, bei 3 Enthaltungen, zu, womit das gemäss Statuten erforderliche Mehr von $3 / 4$ der Stimmen (127) überschritten ist.

170 Delegierte nehmen an der Wahl der Vizepräsidenten teil.

Gewählt sind:

Dr. Ursula Steiner-König (neu)

152 Stimmen

Dr. Yves Guisan (bisher)

143 Stimmen

4.5 Wahl des Präsidenten der Kommission für Weiter- und Fortbildung/KWFB)

159 Delegierte beteiligen sich an der Wahl des KWFBPräsidenten.

Gewählt ist:

Dr. Max Giger (neu)

150 Stimmen

\subsection{Wahl der Finanzkommission der FMH}

Die Wahl erfolgt «in globo», sowohl für die beiden bisherigen Mitglieder, die Dres. 0. Kappeler (TG) und A. Sury (TI) als auch für das neue, von der SMSR vorgeschlagene Mitglied, Dr. Pierre Guillaume (FR). Alle drei sind mit 171 Stimmen gewählt.

\subsection{Wahl des Büros des Schweizerischen Ärztlichen} Ehrenrates (SÄE)

Auch diese Wahl erfolgt "in globo» und per Akklamation, da keine anderen als die vom VEDAG, der SMSR und dem ORDINE vorgeschlagenen Kandidaten zur Verfügung stehen. Es handelt sich um Dr. Sergio Luisoli (bisher), Prof. Markus Heinz Knoblauch (ZH; neu), und Dr. Dominique Haefeli (NE; neu).

NB: Da der bisherige und die beiden neuen Mitglieder noch keine Gelegenheit hatten, sich zu treffen, wird die Wahl des Präsidenten des SÄE auf die nächste Ärztekammersitzung verschoben.
4.8 Beirat Verhandlungen TarMed-Tarifstruktur Der ZV hatte der Ärztekammer die Bildung eines Beirates von 10 Mitgliedern vorgeschlagen, davon 4 für die Foederatio Medicorum Scrutantium (FMS), 4 für das Kollegium für Hausarztmedizin (KHM) und 2 für die Foederatio Medicorum Curantium (FMC).

Ein gemeinsam von der Schweizerischen Gesellschaft für Psychiatrie und Psychotherapie und der Schweizerischen Gesellschaft für Kinder- und Jugendpsychiatrie und -psychotherapie eingereichter Antrag verlangt die Aufstockung dieses Beirates auf 12 Mitglieder, damit 2 Sitze für die SGP zur Verfügung stehen.

In der Abstimmung wird dieser Antrag mit 138 gegen 21 Stimmen und 8 Enthaltungen angenommen.

Gleichzeitig nimmt die Ärztekammer Kenntnis von den Anträgen der FMS, des KHM und der SGP und genehmigt diese stillschweigend und ohne Gegenanträge. Der 12 Mitglieder umfassende Beirat setzt sich demnach wie folgt zusammen:

Für die FMS: Dres: Beat Meister (BE), Alexander Eijsten (ZH), Walter P. Gartenmann (ZH) und Roberto Tartini (ZH). Für den Fall einer Verhinderung eines der Gewählten, hat die FMS folgende Stellvertreter vorgesehen: Fürspr. François A. Bernath und die Dres. Christoph Krayenbühl, Rolf Steiner und Josef Hug;

Für das KHM: Dres. Christoph Francke (SG), Niklaus Hasler (ZH), Juerg Pellaton (ZH) und Emile Simon (VD);

Für die FMC: Dres. Markus Heitz (ZH) und Andreas Wüest $(\mathrm{ZH})$;

Für die SGP: Dres. Heiner Lachenmeier $(\mathrm{ZH})$ und Urs Fromm (AG).

Bevor das Traktandum Wahlen verlassen wird, liegt dem Präsidenten daran, den drei auf das Ende dieser Amtsperiode zurücktretenden Mitgliedern die Ehre zu erweisen und zu danken, indem er ihre Verdienste und die von ihnen in zahlreichen Jahren der Tätigkeit erbrachten Aufgaben im Dienste der FMH und der gesamten Ärzteschaft hervorhebt. Wir werden auf dieses Thema in einem Artikel in der SÄZ zurückkommen. Jedes der zurücktretenden Mitglieder wendet sich anschliessend persönlich an die Versammlung und erntet Applaus von den Delegierten.

\section{Statutenänderungen}

Dr. A. Haefeli nimmt namens des VEDAG seine bereits früher in einem Antrag zu Traktandum Nr. 4.1 aufgeworfene Idee auf, und lädt den ZV ein, seine Zusammensetzung, Struktur und Arbeitsweise zu überprüfen und der Ärztekammer ein entsprechendes Konzept vorzulegen, unter Berücksichtigung einer Reduktion der Anzahl ZV-Mitglieder, einer angemessenen Vertretung der Sprachregionen, der Nicht-Einsitznahme von Interessenvertretern bzw. der Offenlegung von potentiell problematischen Verbindungen, einer Erhöhung des Professionalisierungsgrades, einer aktiveren Rolle des ZV und seines Präsidenten bei der Auswahl von Kandidatinnen und Kandidaten 
und schliesslich, einer mit Rücksicht auf die Umstellung ihrer beruflichen Tätigkeit längerfristigen Vorbereitung der Kandidatinnen und Kandidaten auf ihr künftiges Amt. Vorgesehener Zeitplan: Vorlage einer Projektskizze an der Ärztekammer 2001, detailliertes Konzept im Jahr 2002, damit Kandidatinnen und Kandidaten für den Einsitz in den ZV in der nächsten Amtsperiode genügend früh aufgebaut werden können.

In der Abstimmung wird der Antrag des VEDAG, dem der ZV nicht opponiert, mit 132 Stimmen, einer Gegenstimme und 17 Enthaltungen angenommen.

Der Antrag Nr. 3 zu Traktandum Nr. 5, der namens der Ärztegesellschaft des Kantons Bern von Dr. R. Streit unterbreitet wird, zielt im wesentlichen in dieselbe Richtung wie der VEDAG-Antrag, verlangt aber darüber hinaus «einen erweiterten Vorstand mit umschriebenen Kompetenzen, der zwischen den kleinen Vorstand und die Ärztekammer zwischengeschaltet werden soll".

Nach einer kurzen Debatte, in welcher die Redner sich mehrheitlich skeptisch gegenüber einer solchen Institution äussern, wird abgestimmt. Der Antrag von Dr. R. Streit wird mit 102 zu 49 Stimmen und 21 Enthaltungen abgelehnt.

Ein gemeinsam von SGAM und SGIM eingereichter Antrag Nr. 1 zu Traktandum Nr. 5 verlangt: «Die FMH wird beauftragt, geeignete Kriterien zu formulieren, welche die in den neuen FMH-Statuten verankerte Proportionalität der Sitzverteilung der Fachgesellschaften in der Ärztekammer gewährleisten.»

Dieser Antrag wird genehmigt, mit $134 \mathrm{zu} 25$ Stimmen und 8 Enthaltungen.

Dies veranlasst Frau Dr. A. Kubli, den Antrag Nr. 2 zu Traktandum Nr. 5, der gemeinsam von den beiden Fachgesellschaften für Psychiatrie und Psychotherapie (Erwachsene/Kinder und Jugendliche) eingereicht wurde und dasselbe Ziel anstrebte, zurückzuziehen.

\section{Weiterbildung}

\subsection{Neuordnung Facharzttitel/Weiterbildungs- programme}

\subsubsection{Revision von Facharzttiteln}

Bevor man in die Detailberatung und die Beschlussfassung eintritt, erläutert der abtretende Präsident der KWFB, Dr. R. Salzberg, worum es bei der Debatte und den Beschlüssen geht. Er erinnert daran, dass 1994, als man vor einer Proliferation der Facharzttitel stand, ein Moratorium beschlossen wurde. Daraus resultierte 1997 eine Revision der Weiterbildungsordnung (WBO), die zur Neuschaffung von Facharzttiteln, Schwerpunkten, Fähigkeits- und Fertigkeitsausweisen führte, welche die ehemaligen Subdisziplinen ersetzen und integrieren sollten. Inzwischen sind sowohl neue Facharzttitel als auch Schwerpunkte geschaffen worden. Die Ärztekammer hat eine Usanz entwickelt, wonach eine Weiterbildung höchstens 6 Jahre dauern darf, man sich gegenüber der Schaffung von neuen Titeln restriktiv zeigen will und diese letzteren mindestens 3 Jahre fachspezifische Weiterbildung umfassen müssen. Weiter wurde ein Modell beschlossen, wonach der Facharzttitel plus 3 Jahre fachspezifische Weiterbildung $\mathrm{zu}$ einem Schwerpunkt führen können (z.B. Neonatologie).

An der heutigen Sitzung wird über zwei Problemkreise verhandelt, die sich nicht in dieses erwähnte Konzept einschreiben lassen; es sind dies die Neuroradiologie und die ehemaligen Subdisziplinen der Pädiatrie. Der Redner verweist auf die Grundsätze, welche die Ärztekammer bei ihrer Beschlussfassung zu berücksichtigen hat: Der Facharzttitel ist die Bestätigung für eine strukturierte und kontrollierte Weiterbildung gemäss entsprechendem Weiterbildungsprogramm in einem Fachgebiet der klinischen oder nicht-klinischen Medizin, währenddem, wie der Name besagt, der Schwerpunkt eine Spezialisierung innerhalb eines Fachgebietes darstellt. Die für diese Schwerpunkte verantwortlichen Gruppierungen sind in der KWFB vertreten und können dort ihre Anliegen vorbringen; demgegenüber sind diese Gruppierungen als solche in der Ärztekammer nicht vertreten, sondern müssen ihre Anliegen via ihre Muttergesellschaft einbringen lassen (Beispiel: die Neonatologen müssen die Schweizerische Gesellschaft für Pädiatrie damit beauftragen usw.). Im übrigen müssen wir die entsprechenden Praktiken der EU-Ärzte berücksichtigen und die Art und Weise beachten, wie diese Spezialisierungen dort geregelt sind. Abschliessend ist jede Fachgesellschaft, die einen Facharzttitel führt, verpflichtet, ihr Weiterbildungsprogramm aktuell zu halten und es der medizinischen Entwicklung anzupassen, sie muss eine Facharztprüfung durchführen und Fortbildung wie auch Qualität der Leistungen ihrer Mitglieder garantieren.

Die Ärztekammer ist nur für die Schaffung eines Titels oder eines Schwerpunktes zuständig, während die konkrete Ausgestaltung und die Inkraftsetzung der Programme anschliessend durch den ZV erfolgen. Unterbreitet eine Fachgesellschaft ein Programm für die Schaffung eines Facharzttitels, welches in der KWFB unbestritten war, wird über dieses Programm hier nicht verhandelt. Liegen aber zu Programmen Anträge vor, kommt es zu einer Debatte in der Ärztekammer.

Daraufhin tritt man in die Detailverhandlungen ein.

Mit Antrag Nr. $1 \mathrm{zu}$ Traktandum Nr. 6 verlangt Dr. M. Battaglia namens des VSAO eine Umstellung der Traktandenliste, wonach zuerst die Revision der Weiterbildungsordnung (WBO) (in der Traktandenliste unter Traktandum 6.2 vorgesehen) und erst anschliessend sämtliche Titel und Programme im Rahmen der Neuordnung Facharzttitel/Weiterbildungsprogramme (vorgesehen unter Traktandum Nr. 6.1) zu diskutieren seien. Dr. R. Salzberg widersetzt sich namens des Zentralvorstandes dieser Änderung der Spielregeln.

In der Abstimmung wird der Antrag des VSAO mit $124 \mathrm{zu} 30$ Stimmen und 10 Enthaltungen abgelehnt. 
Die Verhandlungen werden demzufolge gemäss der vom ZV vorgesehenen Tagesordnung durchgeführt. 6.1.1.1 Anästhesiologie

Der Kammer liegt ein Antrag von Dr. M. Battaglia, namens des VSA0, vor, welcher sich einer Verlängerung der Weiterbildung von 5 auf neu 6 Jahre widersetzt.

In der Abstimmung wird der Antrag des Zentralvorstandes (6 Jahre) mit 110 gegen 39 Stimmen und 18 Enthaltungen angenommen, womit folglich der Antrag des VSA0 abgewiesen ist.

6.1.1.2 Plastisch-rekonstruktive und ästhetische Chirurgie

Es sei an dieser Stelle erwähnt, dass dieses Traktandum durch die Fachgesellschaft selbst von der Traktandenliste zurückgezogen wurde, nachdem diese festgestellt hatte, dass die Bezeichnung «ästhetisch", mit welchem sie den Namen des Titels hatte ergänzen wollen, in den entsprechenden ausländischen Titelbezeichnungen auch nicht vorhanden ist.

\subsubsection{Schaffung von Facharzttiteln}

6.1.2.1 Neuroradiologie; Beschluss der WBK vom 11. Mai 2000

Dr. M. Giger, neuer Präsident der KWFB, führt in die Problematik ein, die wir hier zusammenfassend wiedergeben.

Die Meinungsverschiedenheit zwischen der Schweizerischen Gesellschaft für Medizinische Radiologie (SGMR) und der Schweizerischen Gesellschaft für Neuroradiologie besteht darin, dass erstere für die Schaffung von Schwerpunkten für die Neuroradiologie eintritt, während letztere die Bildung eines Facharzttitels für ihre Disziplin verlangt, mit der Begründung (ihr Vertreter Dr. 0. Schubiger erläutert dies vor der Ärztekammer), dass es nicht mehr weiter möglich sei, beide Fachgebiete abzudecken und demzufolge befürchtet werden müsse, dass diese Spezialität ohne einen eigenen Facharzttitel verschwinde. Die KWFB und die Präsidentenkonferenz beantragen bzw. empfehlen der Ärztekammer, die Schaffung zweier Schwerpunkte: «Diagnostische Neuroradiologie» und "Interventionelle Neuroradiologie».

Im Verlaufe der lebhaften Diskussion, in der die Befürworter der beiden Thesen die Klingen kreuzen, bringt Prof. F. Follath (SGIM) einen Antrag ein, wonach beide Gesellschaften nochmals ihre Diskussionen aufnehmen und an einer nächsten Ärztekammer ein Weiterbildungsprogramm vorlegen sollen, welches den Erwerb eines Doppeltitels Facharzt für Radiologie und Facharzt für Neuroradiologie ermöglicht. Dr. M. Giger widersetzt sich diesem Ansinnen, indem er auf die Gefahr aufmerksam macht, die ein solches Ausweichmanöver für eine grosse Zahl anderer Bereiche der radiologischen Weiterbildung (Pädiatrische Radiologie, Radio-Onkologie) darstellen könnte, und bezeichnet es als dringend notwendig, dass die Ärztekammer in dieser Kontroverse heute entscheide. Bevor man zur Abstimmung schreitet, ergreift Prof. P. Schnyder (CHUV) in seiner Eigenschaft als Inhaber eines Lehrstuhls für Radiologie und Schweizer Vertreter in den höchsten europäischen
Instanzen dieses Fachs das Wort und opponiert entschieden dem Antrag der Schweizerischen Gesellschaft für Neuroradiologie. Er macht unter anderem auf die finanziellen Folgen einer Schaffung eines neuen Facharzttitels Neuradiologie für die Weiterbildungsstätten aufmerksam, weist auf die Gefahr einer Spaltung innerhalb des Fachgebietes hin und orientiert, dass zum heutigen Zeitpunkt nur zwei europäische Länder (Schweden und Portugal) einen solchen Titel kennen.

In der Abstimmung wird der Antrag von Prof. F. Follath mit 130 zu 24 Stimmen und 4 Enthaltungen abgelehnt.

Zur Abstimmung gelangt nun der Antrag des ZV. Mit 147 Stimmen beschliessen die Delegierten die Schaffung zweier Schwerpunkte, und damit gleichzeitig die Aufnahme der Schweizerischen Gesellschaft für Neuroradiologie in die KWFB.

\section{Pädiatrische Subdisziplinen}

Auch hier geht es darum, zwischen dem Facharzttitel und dem Schwerpunkt zu entscheiden. Dr. H. H. Brunner präzisiert, dass die getroffene Entscheidung für sämtliche der aufgezählten Subdisziplinen Geltung haben muss und es nicht in Frage kommt, dass einige davon anders als die Übrigen behandelt werden. Er schlägt konsequenterweise vor, dass über alle diese Subdisziplinen «in globo» abgestimmt wird, es sei denn, jemand verlange die Diskussion über eine bestimmte Subdisziplin.

Nach einer Debatte, in welcher die vorliegenden Thesen abwechslungsweise verteidigt werden, insbesondere durch die Vertreter der verschiedenen Gesellschaften im Einzugsbereich der Pädiatrie, die sich für die Schaffung von Facharzttiteln einsetzen, schreitet man zur Abstimmung. Der Präsident stellt vorgängig fest, dass niemand einer Abstimmung «in globo" für alle betroffenen Subdisziplinen opponiert. Die Ärztekammer entscheidet sich zugunsten einer Lösung mit Schwerpunkten, mit 114 gegen 43 Stimmen und 7 Enthaltungen.

Die getroffene Entscheidung hat dementsprechend für die folgenden Subdisziplinen Gültigkeit: 6.1.2.2 Pädiatrische Endokrinologie-Diabetologie

6.1.2.3 Pädiatrische Gastroenterologie

6.1.2.4 Pädiatrische Kardiologie

6.1.2.5 Pädiatrische Nephrologie

6.1.2.6 Pädiatrische Neurologie

6.1.2.7 Pädiatrische Onkologie-Hämatologie

6.1.2.8 Pädiatrische Pneumologie

\section{Andere Weiterbildungsprogramme}

Über die Weiterbildungsprogramme, die hier folgen, wurde nur diskutiert, wenn ein Gegenantrag zum ZVAntrag vorlag (dies war der Fall für Punkt 6.1.2.13; in allen anderen Fällen wurde der entsprechende Antrag des ZV als genehmigt betrachtet. Die Ärztekammer hat diesem Vorgehen nicht opponiert.

Diese Lösung ohne Diskussion wurde auf die folgenden Spezialitäten angewandt:

6.1.2.9 Allergologie und Klinische Immunologie: Schaffung eines Facharzttitels FMH 
6.1.2.10 Arbeitsmedizin: Schaffung eines Facharzttitels FMH

6.1.2.11 Endokrinologie-Diabetologie: Schaffung eines Facharzttitels FMH

6.1.2.12 Hämatologie: Schaffung eines Facharzttitels FMH

6.1.2.13 Intensivmedizin: Die Ärztekammer hat sich hier mit einem Antrag des VSAO zu befassen, welcher die Schaffung eines Facharzttitels ablehnt, weil dies wegen der für das Fachgebiet Intensivmedizin erforderlichen breiten Basisweiterbildung in anderen klinischen Fächern keinen Sinn macht. Dr. R. Stocker, für die Schweizerische Gesellschaft für Intensivmedizin, und Dr. B. Meister (Anästhesiologie) plädieren für die Schaffung des Titels, indem sie auf die respektable Zahl von Intensivmedizinern (250 und 30 in Weiterbildung) und die 60000 spezifischen Interventionen pro Jahr hinweisen und in Sorge sind, das in den 60 intensivmedizinischen Abteilungen tätige Pflegepersonal zu verlieren.

Vor der Abstimmung erklärt der Präsident $\mathrm{H}$. H. Brunner, dass diejenige, welche den Antrag des ZV (Schaffung des Facharzttitels Intensivmedizin FMH) ablehnen, als Befürworter eines Schwerpunktes, entsprechend dem Antrag des VSA0, betrachtet werden.

In der anschliessenden Abstimmung wird der Antrag des ZV mit 78 zu 65 Stimmen und 9 Enthaltungen abgelehnt; daraus folgt, dass sich die Delegierten mehrheitlich für die Schaffung eines Schwerpunktes entschieden haben.

\section{Wichtiger Hinweis:}

Kaum ist das Resultat bekannt, wirft Dr. R. Stocker die Frage der Schwerpunkte auf und macht geltend, dass es unmöglich ist, für 7 verschiedenen Fachbereiche Schwerpunkte zu schaffen. Dr. R. Salzberg anerkennt, dass hier ein echtes Problem besteht. Man wird sich zweifelsohne in der KWFB und auch im ZV nochmals an die Arbeit machen, allenfalls wieder vor die Ärztekammer kommen müssen. Dies zur Kenntnisnahme.

6.1.2.14 Klinische Pharmakologie und Toxikologie: Schaffung eines Facharzttitels FMH

6.1.2.15 Nephrologie: Schaffung eines Facharzttitels FMH

6.1.2.16 Medizinische Onkologie: Schaffung eines Facharzttitels FMH

6.1.2.17 Pneumologie: Schaffung eines Facharzttitels FMH

6.1.2.18 Radiologie: Die Ärztekammer hat hier in einer Meinungsverschiedenheit bezüglich der Weiterbildungsdauer zu entscheiden, 6 Jahre gemäss Antrag des ZV oder nur 5 Jahre, wie dies der VSA0 verlangt. In der Abstimmung siegt der ZV-Antrag mit 100 gegen 40 Stimmen und 14 Enthaltungen.

6.1.2.19 Nuklearmedizin: Schaffung eines Facharzttitels FMH

6.1.2.20 Radio-Onkologie/Strahlentherapie: auch hier muss über eine identische Kontroverse bezüglich Weiterbildungsdauer, zwischen 6 Jahren gemäss An- trag ZV oder 5 Jahren gemäss Antrag VSA0, entschieden werden.

In der Abstimmung siegt der ZV-Antrag mit 116 gegen 25 Stimmen und 11 Enthaltungen. Die Schaffung des Facharzttitels war unbestritten.

\subsubsection{Schaffung von Schwerpunkten}

6.1.3.1 Diagnostische Neuroradiologie (zu Radiologie): Schaffung eines Schwerpunktes und Aufnahme der Fachgesellschaft in die KWFB.

6.1.3.2 Interventionelle Neuroradiologie (zu Radiologie): Schaffung eines Schwerpunktes und Aufnahme der Fachgesellschaft in die KWFB.

6.1.3.3 Pädiatrische Radiologie (zu Radiologie): Schaffung eines Schwerpunktes und Aufnahme der Fachgesellschaft in die KWFB.

6.1.3.4 Reproduktionsmedizin und gynäkologische Endokrinologie (zu Gynäkologie und Geburtshilfe): Diese Angelegenheit reiht sich in einen bestimmten Kontext ein, der an dieser Stelle einiger Erklärungen bedarf. Der Bundesrat muss in Kürze das Bundesgesetz über die Fortpflanzungsmedizin in Kraft setzen, das vom Parlament im Dezember 1998 angenommen wurde. Dieses Gesetz sieht vor, das Ärztinnen und Ärzte, die diese Techniken praktizieren, eine Bewilligung erhalten müssen. Anstatt, dass es der Bund ist, welcher die Anforderungen festlegt, zeichnet sich eine Lösung ab, dass es die FMH sein könnte, welche durch die vorgezogene Schaffung eines Schwerpunkts die Weiterbildungsanforderungen im Rahmen ihrer WBO definiert. Das Bundesamt für Justiz schlägt dem Bundesrat vor, in der Ausführungsverordnung zum Fortpflanzungsmedizingesetz auf die FMH-Regelung zu verweisen. Damit erklärt sich der Druck, unter dem wir in der vorliegenden Angelegenheit stehen.

Dieser Zeitdruck war für den VSAO nicht akzeptabel, weshalb er vorerst, ohne sich grundsätzlich gegen die Schaffung eines Schwerpunktes zu stellen, einen Antrag auf Nichteintreten einreichte. Dieser wird im Laufe der Verhandlungen zurückgezogen, nach der abgegebenen Versicherung, dass das (nicht bestrittene) Programm des Schwerpunktes, welches 1999 im Zusammenhang mit dem Programm für den Facharzttitel Gynäkologie und Geburtshilfe vorgestellt worden war, keinerlei Änderungen erfahren hat.

Die Kammer kann damit den Antrag des ZV annehmen und grundsätzlich einen Schwerpunkt Reproduktionsmedizin und gynäkologische Endokrinologie schaffen. Es wird zur Kenntnis genommen, dass die allenfalls aus diesem vorgezogenen Beschluss sich ergebenden technischen Detailfragen in Bezug auf den noch nicht revidierten Haupttitel, zwischen der betroffenen Fachgesellschaft, der KWFB und dem ZV zu regeln sein werden.

\subsubsection{Schaffung von Fähigkeitsausweisen}

6.1.4.1 Tauchmedizin (SGUHM): Schaffung eines Fähigkeitsausweises Tauchmedizin (SGUHM).

\subsubsection{Schaffung von Fertigkeitsausweisen}

6.1.5.1 Dosisintensives Röntgen: Schaffung eines Fertigkeitsausweises Dosisintensives Röntgen. 
6.1.5.2 Labor (KHM): Schaffung eines Fertigkeitsausweises

6.1.5.3 Laserbehandlung der Haut und der Schleimhäute (FMS): Schaffung eines Fertigkeitsausweises.

\subsection{Revision der Weiterbildungsordnung (WBO)}

Vor der Abstimmung über die gesamte WBO, muss sich die Kammer mit einem Antrag von Dr. M. Battaglia, eingereicht namens des VSA0, befassen. Beantragt wird, dass sich die Rekurskommission aus fünf Mitgliedern, darunter einem unabhängigen Juristen zusammensetzt. Zwei Mitglieder sollen dem ZV, eines einer kantonalen Ärztegesellschaft und ein Mitglied dem VSAO angehören.

Seitens des ZV werden die guten Erfahrungen mit einer aus drei Mitgliedern bestehenden Kommission sowie das Bestreben nach einer kostengünstigeren Verwaltung geltend gemacht.

In der Abstimmung wird der Antrag des $Z V \mathrm{zu}$ diesem konkreten Punkt mit 115 gegen 19 Stimmen und 5 Enthaltungen genehmigt.

Auf Ersuchen von Dr. R. Salzberg stimmt die Kammer nach all diesen gefassten Detailbeschlüssen formell über die WBO in ihrer Gesamtheit $a b$, ungeachtet dessen, dass sie auf den 1. Januar 2001 durch ein Bundesgesetz ersetzt wird.

In der Abstimmung wird die WBO buchstäblich durch Plebiszit angenommen, mit 138 gegen 2 Stimmen und 6 Enthaltungen.

6.3 Freizügigkeitsgesetz: Erteilung von Eidg. Weiterbildungstiteln an Nichttitelträger

Der Gesetzgeber hat im Art. 24 Abs. 3 des Freizügigkeitsgesetzes vorgesehen, allen Nichttitelträgern einen ihrer theoretischen und praktischen Weiterbildung entsprechenden Weiterbildungstitel aus den in der Ausführungsverordnung zum Gesetz aufgeführten Titeln zu erteilen. Diese Bestimmung erweist sich als notwendig, weil eine freie ärztliche Berufsausübung ohne eidgenössischen Titel nicht mehr möglich sein wird. Die Verordnung sieht zwei Varianten vor, über welche der ZV die Ärztekammer konsultativ befragen will, obwohl die endgültige Entscheidung dem Bundesrat zustehen wird.

Variante I, die ihrerseits zwei Subvarianten enthält, postuliert die gesamte Überprüfung der Weiterbildung. In der Subvariante 1 könnte ein fehlendes Weiterbildungsjahr mit einem Jahr Berufsausübung in einer Arztpraxis ersetzt werden, während die Subvariante 2 eine fehlende Weiterbildung von bis zu 3 Jahren durch Kompensation mit ärztlicher Praxistätigkeit zulassen würde.

Variante II, welche die Wünsche der SGAM berücksichtigt, würde die Erteilung des Titels Facharzt für Allgemeinmedizin nur dann erleichtern, wenn der Kandidat zwei Jahre anerkannte Weiterbildung ausweisen kann.

Dr. R. Hohendahl von der Foederatio Medicorum Practicorum (FMP) beantragt der Ärztekammer, den ZV zu beauftragen (mit dem BAG) weiterhin im Sinne der Subvariante $2 \mathrm{zu}$ verhandeln.
Nach einer lebhaften Debatte, kommt der Antrag der FMP zur Abstimmung: er wird mit 91 gegen 37 Stimmen und 9 Enthaltungen abgelehnt.

\section{Aufnahme der Chiropraktorengesellschaft als \\ 4. Gründerverein der AHV-Ausgleichskasse der Ärzte, Zahnärzte und Tierärzte (AHV-Kasse Nr. 28)}

Dieses Traktandum wird in Anwesenheit von Franziska Iseli, Notarin in Bern, behandelt.

Der Generalsekretär erläutert kurz nochmals die Hintergründe des Antrages. Das Wort dazu wird nicht verlangt.

Anschliessend stellt der Vorsitzende im Namen des Zentralvorstandes der FMH folgenden Antrag: «Der Zentralvorstand beantragt der Schweizerischen Ärztekammer, dem Gesuch der Chiropraktorengesellschaft um Aufnahme als 4. Gründerverein in die AHV-Ausgleichskasse Nr. 28 ab dem 1. Januar 2001 zuzustimmen."

Der Antrag erhält folgende Stimmen: $161 \mathrm{Ja}$, 0 Nein, 2 Enthaltungen. Die gemäss Art. 53 AHVG erforderliche Mehrheit von $3 / 4$ der abgegebenen Stimmen (123) ist erreicht und der Antrag ist damit angenommen.

\section{Standesordnung FMH/Partialrevision}

In einem von Prof. L. von Laer namens der Medizinischen Gesellschaft Basel eingereichten Antrag wird verlangt, dass der unter Art. 47 Abs. 1 der FMH-Standesordnung aufgeführte Sanktionskatalog auf den 1. Januar 2001 ergänzt wird mit der Möglichkeit, die betroffenen Ärztinnen und Ärzte durch das Mittel der Supervision (lit. h neu) zu ermutigen und zu unterstützen. Der Antragsteller macht geltend, dass auf diese Weise in gewissen Fällen (sexueller Missbrauch von Patientinnen und Patienten) eine bessere und dauerhaftere Wirkung erreicht werden kann als mit reinen Strafen.

Der Präsident H. H. Brunner opponiert diesem Antrag nicht, der von der Ärztekammer stillschweigend genehmigt wird. Er wird zur Ausführung an die kompetenten Stellen weitergeleitet.

\section{Heilmittelgesetz (mündliche Information)}

Mit dem Gesetz, welches im Nationalrat behandelt worden ist, ohne dass unsere Wünsche berücksichtigt wurden, befasst sich zur Zeit die ständerätliche Adhoc-Kommission: hier müssen wir also unsere Anliegen (freiheitliche Lösung der Medikamentendistribution, indem man damit alle Personen betraut - Ärztinnen und Ärzte eingeschlossen - welche über die notwendige Kompetenz verfügen, sowie verantwortliche Lösung für die Sterilisation). Es sei an dieser Stelle erwähnt, dass die Ärztekammer dem ZV die Kompetenz übertragen hatte, sofern notwendig, eine 
Volksinitiative zu lancieren. Im übrigen bleibt uns ebenfalls die Möglichkeit eines Referendums gegen das Gesetz. Dr. A. Haefeli schlägt zudem vor, dass der ZV zuhanden der Kantonalen Ärztegesellschaften ein Papier erstellt, welches die aus Sicht der FMH unbedingt in das Gesetz gehörenden Punkte zusammenfasst; der Präsident H. H. Brunner nimmt davon im Namen des ZV Kenntnis. Die Angelegenheit wird weiter verfolgt.

\section{Kontrahierungszwang (mündliche Information)}

Dr. R. Streit verlangt mit einem Antrag namens der Ärztegesellschaft des Kantons Bern zum Traktandum $N r .10$, dass «eine Grundsatzdebatte über die gesundheitspolitische Situation zu führen sei, unter Berücksichtigung der Erklärung von Bundesrätin Dreifuss im Ständerat, wonach der Taxpunktwert etwa 80 Rappen für TarMed betragen werde, und über den Antrag des Eidg. Departementes des Innern auf Aufhebung des Kontrahierungszwanges in der sozialen Grundversicherung." Dieser Antrag wir praktisch einstimmig mit einzelnen Gegenstimmen angenommen.

Es folgt eine lange Debatte, die, wie wenn es dessen noch bedürft hätte, den Grad der Erbitterung aufzeigt, die unter der innerhalb der FMH vereinigten Ärzteschaft herrscht. Scharfe und heftige Worte werden an die Adresse von Bundesrätin Dreifuss gerichtet wegen ihrer neusten Druckversuche auf unsere Verhandlungen und weil sie aus der Zurückhaltung, die sie als künftige "Richterin in letzter Instanz" in Sachen TarMed zu beachten hätte, in einem solchen Masse herausgetreten ist, dass sie in den Augen der Ärztekammer jede Glaubwürdigkeit in ihre Unparteilichkeit verloren hat.

Was den Kontrahierungszwang im besonderen betrifft, ist die Kammer bereit, sich über eine Einschränkung des Kontrahierungszwanges zu verständigen, mit dem Ziel, die besten Ärztinnen und Ärzte im Dienste der Patientinnen und Patienten der Sozialversicherung arbeiten zu lassen, und erinnert an ihre diesbezüglichen Vorschläge an das BSV und das Konkordat der Schweizerischen Krankenversicherer (KSK). Demgegenüber würde die FMH nicht vor einem Referendum zurückschrecken, wenn die Gesetzesreform so herauskäme, wie sie vom Eidg. Departement des Innern vorgeschlagen wird.

Betreffend TarMed und ohne die noch unter Traktandum Nr. $11 \mathrm{zu}$ fassenden Beschlüsse zu präjudizieren, erteilen die Delegierten der Verhandlungsdelegation den Auftrag, in den Schlussverhandlungen keinen Taxpunktwert unter Fr. 1.- zu vereinbaren.

Zum Abschluss der Debatte fasst der Präsident H. H. Brunner die wichtigsten Punkte zusammen und informiert die Kammer, dass es darüber ein Pressecommuniqué geben werde. Die Kammer akzeptiert diskussionslos sowohl die vom Präsidenten vorgenommene Zusammenfassung als auch das Prinzip eines Communiqués selbst.

NB: Dieser Entscheid wurde noch am selben Abend in Form der Publikation von zwei Pressecom- muniqués, «Ärzteschaft gegen Pressionsversuche aus dem EDI" und "Aufhebung des Kontrahierungszwangs: FMH diskutiert Referendum», umgesetzt.

\section{TarMed}

Der Präsident H. H. Brunner muss gegenüber den Vertretern der FMS (Dr. C. Krayenbühl und Fürspr. F. A. Bernath) Rede und Anwort stehen, zu verschiedenen Fragen bezüglich der Feldversuche (man hat auf Pilotversuche wegen der voraussehbaren hohen Kosten verzichtet), der Warenkorbstudien (diese müssen ein internes Steuerungsinstrument bleiben) sowie der unabhängigen Rechtsgutachten, betreffend Rechte und Pflichten der diversen Intervenienten bei der Einführung von TarMed (Zögern von kompetenten Juristen, sich zu engagieren und nur mässiges Interesse innerhalb der TarMed). Bessere Aussichten bestehen für ein Mandat, welches einem neutralen ausländischen Institut übertragen werden soll, zur Frage der Erstellung der Tarifstruktur nach betriebswirtschaftlichen Grundsätzen.

Die Fragesteller/Antragsteller erklären sich mit den Antworten zufrieden.

\section{1 Überarbeitungsrunde 2000}

Die Kammer nimmt vom Ergebnis der Überarbeitungsrunde 2000 sowie vom entsprechenden Kommentar Kenntnis, der sich kaum für eine Darstellung in der SÄZ, mit andern Worten: in der Öffentlichkeit, eignet.

\subsection{FMS-Tarifmodell}

Die Kammer nimmt ebenfalls Kenntnis vom diesbezüglichen schriftlichen Kommentar sowie von den Erläuterungen des Präsidenten im Verlaufe der Debatte, Erläuterungen, die einer Antwort auf den Antrag der FMS (Nr. 2 zu Traktandum Nr. 11.2) gleichkommen, die das FMS-Tarifmodell noch vor Inkrafttreten des TarMed implementieren will.

\subsection{Tarifstruktur TarMed Alpha 3.0 / \\ Absichtserklärung}

Es sei hier festgehalten, dass die Version Alpha 3.0 der Tarifstruktur in Erarbeitung ist. Sie basiert auf der Runde 2000 und enthält alle Anträge und Änderungen, die bis am 27. Mai 2000 behandelt worden sind. Diese Version wird die Verhandlungsbasis für den UV/MV/IV-Arzttarif, den Spitalleistungskatalog sowie die kantonalen Tarife darstellen. Eine Version Alpha 3i wird später publiziert werden; sie wird ihrerseits nur formale und redaktionelle Änderungen berücksichtigen. Sämtliche FMH-Mitglieder werden diese Version, die Gegenstand der Urabstimmung sein wird, auf CD-ROM erhalten. Die Anträge, die uns nach dem 15. April 2000 zugegangen sind, werden auf die erste Überarbeitung der Version 3.0 verschoben.

Mit Antrag Nr. 2 zu Traktandum Nr. 11.3 wollen die Schweizerische Gesellschaft für Psychiatrie und Psychotherapie sowie die Schweizerische Gesellschaft für Kinder- und Jugendpsychiatrie und -psychothe- 
rapie den $\mathrm{ZV}$ beauftragen, "geeignete Massnahmen zu ergreifen, damit den ursprünglichen Zielsetzungen von GRAT Nachachtung verschafft wird, nämlich der besseren Gewichtung der ärztlich-intellektuellen und emotionellen Leistungen [und] der Verringerung der nicht zu rechtfertigenden Einkommensunterschiede zwischen den verschiedenen Fachgesellschaften". Nach der Begründung durch die Antragsteller und der Antwort des Präsidenten H. H. Brunner, wird dieser Antrag von den Delegierten stillschweigend genehmigt.

Ein weiterer Antrag (Nr. 3 zu Traktandum Nr. 11.3), gemeinsam eingereicht von der SGAM, der SGIM, der SGP und dem Kollegium für Hausarztmedizin, verlangt, dass "Ziffer 21 der Generellen Interpretationen zu TarMed Version Alpha 2.2 (oder je nach Version Ziffer 85) zwingend abzuändern sei». Der Antrag, der zu detailliert ist, um hier in extenso wiedergegeben zu werden, zielt im wesentlichen darauf ab, dass die Möglichkeit beibehalten wird, gewisses Verbrauchsmaterial, das in den Arztpraxen der Grundversicherer verwendet wird, zusätzlich zu verrechnen, weil sonst zahlreiche sinnvolle und kostensparende Leistungen nicht mehr in der Sprechstunde erbracht, sondern der Patient ins Spital überwiesen würde. Konkrete Beispiele für solche Aberrationen könnten zuständigen Personen geliefert werden. Die Angelegenheit ist von Wichtigkeit, die Intervenienten seitens der antragstellenden Gesellschaften lassen verlauten, dass eine Aufrechterhaltung der kritisierten Interpretation zu einer negativen Beeinflussung der Urabstimmung über den TarMed führen könnte. Dies wird zur Kenntnis genommen.

\subsection{Rahmenvertrag KSK-FMH}

Nachdem Dr. A. Haefeli, Präsident der «G 7» die Aktivitäten dieser Arbeitsgruppe und die bereits erreichten Zwischenergebnisse präsentiert hat, und nach ausgedehnter Debatte, nimmt die Ärztekammer, gemäss Antrag des $Z V$, Kenntnis vom Rahmenvertrag KSK-FMH und genehmigt ihn stillschweigend. Sie erteilt der Verhandlungsdelegation den Auftrag, "die Verhandlungen aufgrund der zustimmenden Kenntnisnahme und der Beschlüsse der Ärztekammer zu Ende zu führen und den definitiven Entwurf des Rahmenvertrages an der nächsten Ärztekammer vorzulegen». Es sei an dieser Stelle auf ausdrückliches Ersuchen von Dr. L. Dubs der Schweizerischen Gesellschaft für Orthopädie darauf hingewiesen, dass die Bezeichnung EBM in diesem Zusammenhang nicht Evidence-based Medicine bedeutet, sondern "Einheitliche Bemessungsmassstäbe». Dies zur Kenntnisnahme.

\subsection{Kostenneutralitätskonzept KSK-FMH}

Der Antrag des ZV zu diesem Traktandum zielt darauf ab, dass die Kammer vom Konzept Kostenneutralität (KN) KSK-FMH zustimmend Kenntnis nimmt, gegebenenfalls Beschlüsse der Ärztekammer integriert und die Verhandlungsdelegation damit beauftragt, die Verhandlungen zu Ende zu führen und den definitiven Entwurf des Kostenneutralitätskonzeptes an der nächsten Ärztekammer vorzulegen. Es liegen verschiedene Anträge zu diesem Thema vor. Der erste stammt von Dr. R. Streit, Ärztegesellschaft des Kantons Bern, und ist in 5 Punkte gegliedert. Punkt 1 stellt fest, dass die KN, aufgeteilt in Teilbereiche (kantonale, ambulante, spitalambulante und stationäre), die in sich kostenneutral sein sollen, mit dem gesetzlich vorgeschriebenen betriebswirtschaftlichen Tarif nicht vereinbar ist. Dies ist klar und scharf zu kommunizieren. Punkt 2 verlangt eine klare Definition und Abgrenzung der KN. Die Bezeichnung KN bedeutet, dass die Kosten je Versicherten durch die Einführung allein der Struktur nicht verändert werden sollen. Alle tarifwirksamen, nicht strukturbedingten Einflüsse müssen, soweit möglich, erfasst und der KN-Summe positiv oder negativ zugerechnet werden. Punkt 3 betrifft den Kostensteigerungswinkel Alpha: er wird eidgenössisch als Durchschnittswert festgelegt, muss aber in den einzelnen Kantonen angepasst werden, da sich Verschiebungen zwischen kostenneutralen Bereichen von einem Kanton zum anderen ergeben können. Mit Punkt 4 soll die Einpendelungsphase nach Einführung von TarMed auf maximal 2 Jahre beschränkt werden. Schliesslich wird unter Punkt 5 verlangt, dass die vorgelegten Konzepte zu überarbeiten und auf Widersprüche zu prüfen sind.

In der Abstimmung werden die in den Punkten 1, 2, 3 und 5 festgehaltenen Forderungen jeweils mit überwältigendem Mehr angenommen. Punkt 4 wird, was die KN im engeren Sinne betrifft, angenommen, der Präsident gibt jedoch zu beachten, dass die Ärztekammer kurz zuvor im Grundsatz einer Einpendelungsphase von 5 Jahren zugestimmt hat. Der zweite Antrag (Nr. 3 zu Traktandum Nr. 11.5), ebenfalls von Dr. R. Streit (BE), umfasst drei Punkte. Er erinnert die Ärztekammer daran, dass sie einer Tarifstruktur, kalkuliert auf der Basis eines Taxpunktwertes von Fr. 1.zugestimmt hat und deshalb ein Taxpunktwert von Fr. -.80 ausdrücklich abzulehnen ist (Punkt 1), dass Bundesrätin Dreifuss mit ihrer Verlautbarung vor dem Ständerat zum Taxpunktwert vor Vorliegen des Verhandlungsergebnisses sich als gegenüber der Ärzteschaft voreingenommen deklariert hat (Punkt 2) und dass keine abschliessende Zustimmung zu Verhandlungsergebnissen erfolgen soll, solange sie ihre Bemerkungen nicht zurücknimmt (Punkt 3). In der Abstimmung wird dieser Antrag mit sehr grosser Mehrheit angenommen.

Den dritten Antrag (Nr. 4 zu Traktandum Nr. 11.5) hat die Ärztegesellschaft des Kantons Freiburg eingereicht. Er verlangt im wesentlichen, dass die Ärztekammer die Einführung von TarMed nur akzeptiert, wenn das Konzept KN von allen Vertragsparteien (KSK, Medizinaltarifkommission UVG, SDK und $\mathrm{H}+$ ) unterzeichnet ist, weil es Bedingung sein muss, dass nur eine einzige Berechnungsgrundlage und -analyse angewendet wird.

Ein vierter Antrag (Nr. 5 zu Traktandum Nr. 11.5), eingebracht durch die FMS, wird zugunsten der Anträge der Dres. R. Streit und E. Steinmann zurückgezogen. Der Antrag des letzteren (Nr. 6 zu Traktandum Nr. 11.5), eingereicht namens der Ärztegesellschaft 
des Kantons Luzern, fusst auf früheren Ärztekammerbeschlüssen und verlangt, dass das Konzept KN folgende Punkte erfüllen muss: Das Ziel des Konzeptes $\mathrm{KN}$ ist eine kostenneutrale Einführung des neuen Tarifs. Die Phase der strikten KN ist zeitlich minimal zu halten; der Winkel Alpha muss inhaltlich so definiert sein, dass er in Zukunft nicht zu einem Verhandlungsspielball wird; Prinzip, Ziel und Mechanik der Konvergenz (die spätestens nach 5 Jahren abgeschlossen sein muss), sind im Konzept verbindlich zu regeln; die Rahmenbedingungen zur Umsetzung des Konzeptes (Analysenliste, Physiotherapietarif, Regelung der Medikamentenabgabe, Kontrahierungszwang, u.a.) sind integraler Bestandteil des Konzeptes; die Rechtswege bzw. die Forderung nach einem dringlichen Bundesbeschluss fallen nicht in die Kompetenz der Vertragspartner und können somit nicht Inhalt dieses Konzeptes sein. Das vorliegende Grobund Detailkonzept muss deshalb so geändert werden, dass es diesen Forderungen gerecht wird. Der Präsident H. H. Brunner insistiert, dass die Rahmenbedingungen, die eine Garantie für das Funktionieren dieses Konzeptes darstellen, umfassend aufgeführt werden. Dieser Wink an die Politiker sei unabdingbar. Anschliessend muss er dem Absatz bezüglich des dringlichen Bundesbeschlusses opponieren, weil die Dinge bereits in der neusten Version des Konzeptes (es befindet sich noch nicht im Besitze der Delegierten) geregelt sind.

Der Präsident hält zusammenfassend fest, dass der Antrag von Dr. E. Steinmann, mit der abgegebenen Präzisierung durch Dr. H. H. Brunner selbst, stillschweigend akzeptiert ist.

\subsection{Dignitätskonzept}

Die Ärztekammer stimmt dem Antrag des ZV zu, wonach das Dignitätskonzept in der Version 4.6.2000, in einer Version also, die sich nicht auf die aktuelle WBO bezieht, zu genehmigen sei. Der Präsident präzisiert jedoch, dass nichts dagegen stehe, die Zuordnungskriterien der qualitativen Dignität zu einem späteren Zeitpunkt mit den Fachgesellschaften nochmals zu diskutieren.

\subsection{Rahmenvertrag UV/MV/IV}

Die Ärztekammer stimmt dem Antrag des ZV zu, den Rahmenvertrag UV/MV/IV in der Version 4 zu genehmigen.

\subsection{Kostenneutralitätskonzept UV/MV/IV}

Kein Beschluss, da noch kein Papier vorliegt.

\subsection{Start-Taxpunktwert UV/MV/IV}

Die Ärztekammer nimmt vom diesbezüglichen Kommentar Kenntnis; der definitive Beschluss wird an der nächsten Ärztekammer zu treffen sein. Dr. B. Meister, Schweizerische Gesellschaft für Anästhesiologie, verlangt, dass über seinen Antrag, der den Start-Taxpunktwert auf Fr. 1.- festlegen will, abgestimmt wird. In der Abstimmung erhält der Antrag von Dr. B. Meister 125 Ja-Stimmen, 3 Nein-Stimmen und 13 Enthaltungen.
Die Genehmigung dieses Zeitplanes wird allenfalls die Einberufung einer Ärztekammer zur Folge haben, deren entsprechend vorangehende Präsidentenkonferenz nicht statutenkonform 6 Wochen vorher wird stattfinden können. Der Präsident macht die Ärztekammer klar darauf aufmerksam, dass man damit gezwungenermassen gegen die Statuten verstösst. Es wird ausdrücklich festgehalten, dass diesem Vorgehen keinerlei Opposition erwächst.

\subsection{Zeitplan für die Einführung der TarMed-} Tarife

Die Ärztekammer genehmigt mit 138 Stimmen, ohne Gegenstimmen und ohne Enthaltungen, den vom ZV vorgeschlagenen Zeitplan, welcher vorsieht, dass die Verhandlungen so zu führen sind, dass der UV/ MV/IV-Tarif zwischen dem 1. Januar 2001 und dem 1. Juli 2001 eingeführt werden kann. Im besonderen ist/sind der/die Start-Taxpunktwert/e UV/MV/IV bis Ende August 2000 auszuhandeln. Die Arbeiten und Verhandlungen betreffend Einführung von TarMed in den kantonalen Tarifen sind so zu gestalten, dass eine Einführung regulär auf den 1. Juli 2001 möglich ist. Der ZV arbeitet ein Alternativszenario aus, das bei entsprechender Entwicklung des politischen Umfeldes eine Einführung auch per 1. Januar 2001 erlauben würde. Die Rahmenverträge UV/MV/IV mit Anhängen, wie auch KSK-FMH mit Anhängen, sind einer a.o. Ärztekammer zum definitiven Beschluss zu unterbreiten. Der ZV legt das diesbezüglich Datum so fest, dass eine Urabstimmung gewährleistet ist.

\subsection{Urabstimmung}

Die Ärztekammer genehmigt mit 137 Stimmen, ohne Gegenstimmen und ohne Enthaltungen, den Antrag des $Z V$, wonach vor Inkraftsetzung der TarMed-Tarife im UV/MV/IV-Bereich wie auch im KVG-Bereich eine Urabstimmung durchzuführen ist. Gegenstand der Urabstimmung werden sein: die Tarifstruktur TarMed Alpha 3i, der Rahmenvertrag UV/MV/IV mit Anhängen und der Rahmenvertrag KSK-FMH mit Anhängen. Die Urabstimmung wird nur stattfinden, wenn alle diese Punkte von der Ärztekammer genehmigt worden sind. Sie wird unmittelbar nach der Ärztekammer durchgeführt, welche diese entsprechenden Beschlüsse fasst.

\subsection{Elektronischer Datentransfer}

Unter diesem Traktandum sind keine Beschlüsse zu fassen.

\section{PR-Konzept der FMH}

Angesichts der vorgerückten Zeit kann das aus der Feder des Präsidenten stammende Papier nicht diskutiert werden. Letzterer lädt die Delegierten ein, ihm allfällige Bemerkungen und Anregungen, die eine angemessen Berücksichtigung finden werden, zur Kenntnis zu bringen. 


\section{Verschiedenes}

Unter dieser Rubrik ist zu erwähnen:

- dass die FMH in bezug auf die "Call Centers" nicht untätig bleibt und ihre Position zu gegebener Zeit bekannt geben wird.

- dass der ORDINE, vertreten durch Dr. A. Sury, die Ärztekammer einlädt, zum Anlass des 100jährigen Jubiläums der FMH ihre ordentliche Sitzung nächstes Jahr im Tessin durchzuführen. Der Präsident H. H. Brunner nimmt dieses Angebot, das allerdings noch überdacht werden muss, in positivem Sinne entgegen.
Es ist gegen 17 Uhr, als der Präsident die Sitzung schliesst, indem er den Delegierten seine Anerkennung ausspricht für ihre Ausdauer, eine solch beeindruckenden Traktandenliste durchzuberaten, und indem er allen Mitarbeiterinnen und Mitarbeitern des Generalsekretariates - mit besonderer Erwähnung der Übersetzungsabteilung und der Simultanübersetzerinnen - die zum guten Ablauf dieser zwei Sitzungstage beigetragen haben, seinen Dank ausspricht. 